\title{
Nistagmo vertical hacia abajo
}

\author{
Downbeat nystagmus
}

Pablo Cantillano P1, Karen García C'1, Diego Olmedo G², Paul Délano $\mathbf{R}^{1,2,3}$.

\begin{abstract}
RESUMEN
El downbeat nystagmus o nistagmo vertical hacia abajo es el nistagmo de fijación adquirido más frecuente, que en la mayoría de los casos es causado por patología a nivel del sistema nervioso central que genera disrupción del control inhibitorio del flóculo y paraflóculo cerebeloso sobre los núcleos vestibulares. Entre sus causas se encuentran enfermedades neurodegenerativas y vasculares de cerebelo o tronco cerebral, tumores y traumas, pero cerca del $40 \%$ de los casos son idiopáticos y hasta la mitad de los pacientes presentan estudio imagenológico negativo. En este artículo presentamos dos casos que consultaron en el Servicio de Otorrinolaringología del Hospital Clínico de la Universidad de Chile.
\end{abstract}

Palabras claves: Downbeat nystagmus, oscilopsia, vértigo central.

\begin{abstract}
Downbeat nystagmus is the most frequent acquired fixation nystagmus and it is generally caused by central pathology disrupting the inhibitory control of the cerebellar flocculus and paraflocculus over the vestibular nuclei. Among its causes are neurodegenerative and vascular diseases of cerebellum or brainstem, tumors and trauma, but nearly $40 \%$ of the cases are idiopathic and up to half the patients have negative imaging study. In this article we present two cases that were evaluated in the Otolaryngology Department of the Clinical Hospital of the University of Chile.
\end{abstract}

Key words: Downbeat nystagmus, oscillopsia, central vertigo.

\footnotetext{
1 Departamento de Otorrinolaringología, Hospital Clínico de la Universidad de Chile, Santiago, Chile.

2 Servicio de Otorrinolaringología, Hospital Clínico de la Universidad de Chile, Santiago, Chile.

3 Departamento de Neurociencia, Facultad de Medicina, Universidad de Chile, Santiago, Chile.
}

Los autores declaran no tener conflictos de interés. P.D.R. es financiado por CONICYT BASAL FB008, Proyecto ICM P09-015F y Fundación Guillermo Puelma.

Recibido el 17 de octubre, 2018. Aceptado el 18 de mayo, 2019. 


\section{INTRODUCCIÓN}

El nistagmo vertical hacia abajo 0 downbeat nystagmus (DBN) corresponde al nistagmo de fijación adquirido más común ${ }^{1}$, siendo su edad media de presentación cerca de los 60 a 70 años y por lo general traduce patología del sistema nervioso central ${ }^{2}$. Los pacientes se quejan habitualmente de oscilopsia vertical, trastornos del equilibrio estático y de la marcha, además de disminución de la agudeza visual secundaria a la oscilopsia ${ }^{1-3}$. El DBN se caracteriza por ser un nistagmo de fase rápida vertical hacia abajo, persistente e inagotable, que no suprime con la fijación visual e incluso puede aumentar con ésta ${ }^{1}$. Su intensidad puede aumentar, además, con mirada lateral, hacia abajo, convergencia y con menos frecuencia con mirada hacia arriba. La posición en decúbito (prono o supino) también aumenta la intensidad del nistagmo. De forma concomitante los pacientes pueden presentar otras alteraciones oculomotoras y ataxia ${ }^{1-3}$.

Su origen etiopatológico sería una alteración de la actividad inhibitoria gabaérgica de las neuronas de Purkinje del flóculo y paraflóculo del cerebelo, sobre las neuronas de los núcleos vestibulares, especialmente del núcleo vestibular superior ${ }^{1,2,4}$. Diversas lesiones podrían explicar la fisiopatología del DBN: en condiciones normales el flóculo y paraflóculo del vestíbulo-cerebelo generan inhibición sobre el núcleo vestibular superior, específicamente en las vías de los canales semicirculares anteriores, que hacen que los ojos tiendan a moverse hacia arriba. Otro mecanismo que puede generar downbeat nystagmus es la lesión de las neuronas del tracto paramediano que proyectan al flóculo cerebeloso y que reciben importante información desde el canal semicircular anterior ${ }^{5}$. El daño a nivel del flóculo-paraflóculo del cerebelo 0 del tracto paramediano del tronco encefálico desinhibe la vía del control de la mirada superior generando un movimiento ocular lento hacia arriba que se compensa con una fase rápida hacia abajo, generando un downbeat nystagmus ${ }^{4,5}$ (Figura 1). Es importante destacar que el flóculo ejerce una acción inhibitoria sobre la vía del canal semicircular anterior, pero no tiene efecto sobre la vía del canal posterior $r^{4,5}$.

Otras regiones del cerebelo, el nódulo y la úvula ventral, participan en la integración central de la información proveniente de los órganos otolíticos,
Figura 1. Vías implicadas en la fisiopatología del nistagmo vertical hacia abajo. MRS: músculo recto superior, MRI: músculo recto inferior, NCIII: núcleo oculomotor, FLM: fascículo longitudinal medial, CSCA: canal semicircular anterior, NVS: núcleo vestibular superior, TPM: tracto paramediano. Flechas rojas (continuas): vías excitatorias; flechas azules (segmentadas): vías inhibitorias. La lesión cerebelosa a nivel del flóculo/ paraflóculo o del tronco cerebral a nivel del tracto paramediano provoca desinhibición del control de la vía del recto superior, lo que resulta en un downbeat nystagmus.

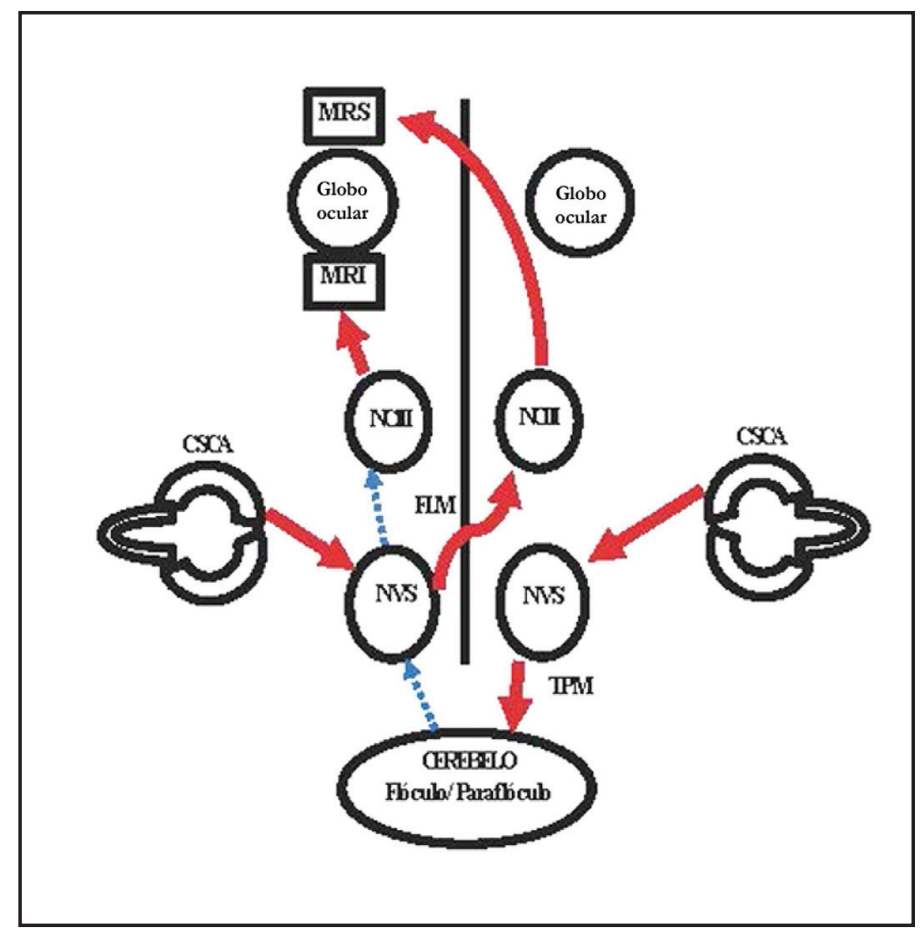


las que al dañarse también pueden generar DBN y explicaría la dependencia de este nistagmo a la posición de la cabeza (aumento en decúbito). De forma menos frecuente otras lesiones de estructuras del sistema nervioso central también pueden generar DBN: el núcleo prepósito hipogloso, el núcleo vestibular medial y el núcleo intersticial de Cajal, los que juegan un rol importante en mantener el ojo estable en mirada excéntrica. Además, lesiones en el sistema de seguimiento lento vertical contribuirían a la fisiopatología de este cuadro.

A nivel central existen vías distintas para el movimiento ocular hacia arriba y abajo, que pueden dañarse en forma selectiva, provocando downbeat 0 upbeat nystagmus: las lesiones del primero ya han sido descritas, y las que provocan upbeat nystagmus se producen a nivel del bulbo caudal (núcleos perihipoglosos como el intercalado de Staderino y de Roller) o lesiones más rostrales que interrumpen proyecciones desde el núcleo vestibular relacionadas con información del canal semicircular posterior ${ }^{4,6,7}$. Del punto de vista clínico, las lesiones del fascículo longitudinal medial se asocian a oftalmoplejía internuclear y pueden presentar DBN de forma unilateral, mientras que las lesiones del tracto paramediano producen DBN bilateral y no necesariamente se asocian a oftalmoplejía internuclear, dependiendo de la indemnidad del fascículo longitudinal medial6-8.

Las causas más frecuentes de DBN son procesos degenerativos y daño vascular en cerebelo 0 tronco encefálico, malformaciones (Arnold-Chiari), tóxicos, procesos inflamatorios, neoplásicos y paraneoplásicos, enfermedades desmielinizantes, hipovitaminosis B12, entre otros. No obstante, solo en $62 \%$ de los casos es posible identificar la causa, mientras que el 38\% restante será catalogado como idiopático ${ }^{2,3}$. A continuación, se presentan dos casos clínicos de DBN que consultaron en el Servicio de Otorrinolaringología del Hospital Clínico Universidad de Chile.

\section{CASOS CLÍNICOS}

\section{Caso 1}

Hombre de 78 años, con antecedentes de infarto agudo al miocardio y usuario de desfibrilador.
Consulta en otorrinolaringología por un año de marcha inestable que lo ha llevado a caídas en tres ocasiones. Además, refiere oscilopsia vertical que empeora con los movimientos cefálicos. No refiere hipoacusia y fue manejado previamente con rehabilitación vestibular sin éxito. Acude con examen de VIII par que muestra hipoacusia sensorioneural bilateral compatible con presbiacusia y prueba calórica con respuestas simétricas. Además, una tomografía computarizada de cerebro sin contraste que evidencia lesión parenquimatosa en cerebelo compatible con secuela de evento isquémico.

Al examen físico destaca DBN, marcha inestable con caída a izquierda, sin dismetría ni disdiadococinesia. Se solicita nuevo VIII par con videonistagmografía (VNG) que objetiva el DBN de $3,4^{\circ}$ por segundo $(\%)$ en su componente vertical hacia abajo, el cual se intensifica con fijación visual y mirada lateral izquierda (Figura 2).

Se inicia tratamiento con pregabalina $(75 \mathrm{mg}$ cada 12 horas por 1 mes), pero debe suspenderse a los diez días por mala tolerancia. Se indica rehabilitación vestibular y oculomotora con estímulo optokinético en planos yaw, pitch y roll, ejercicios de movimientos sacádicos y de supresión del reflejo vestíbulo-ocular. A los tres meses de iniciada la rehabilitación el paciente refiere mejoría sintomática con persistencia del nistagmo y trastorno de la marcha (aumento de base de sustentación, dificultad para marcha en tándem) ante lo cual se agrega terapia kinésica para prevención de caídas.

\section{Caso 2}

Mujer de 65 años derivada para nasofibroscopía en contexto de estudio de disfonía. Durante la consulta en nuestro centro se pesquisa nistagmo espontáneo vertical y al interrogatorio dirigido la paciente refiere estudio previo hace 18 meses por cuadro de oscilopsia progresiva y desequilibrio, sin vértigo ni hipoacusia, acompañado de disminución de la agudeza visual al caminar y levantarse.

Al examen físico se pesquisa marcha atáxica y DBN que aumenta con mirada lateral derecha e izquierda. Se realiza VIII par con VNG que muestra hipoacusia compatible con presbiacusia, y prueba calórica simétrica. El examen oculomotor evidencia DBN de $15,7^{\circ}$ s en posición neutra de la mirada, que aumenta con fijación visual y mirada lateral de- 


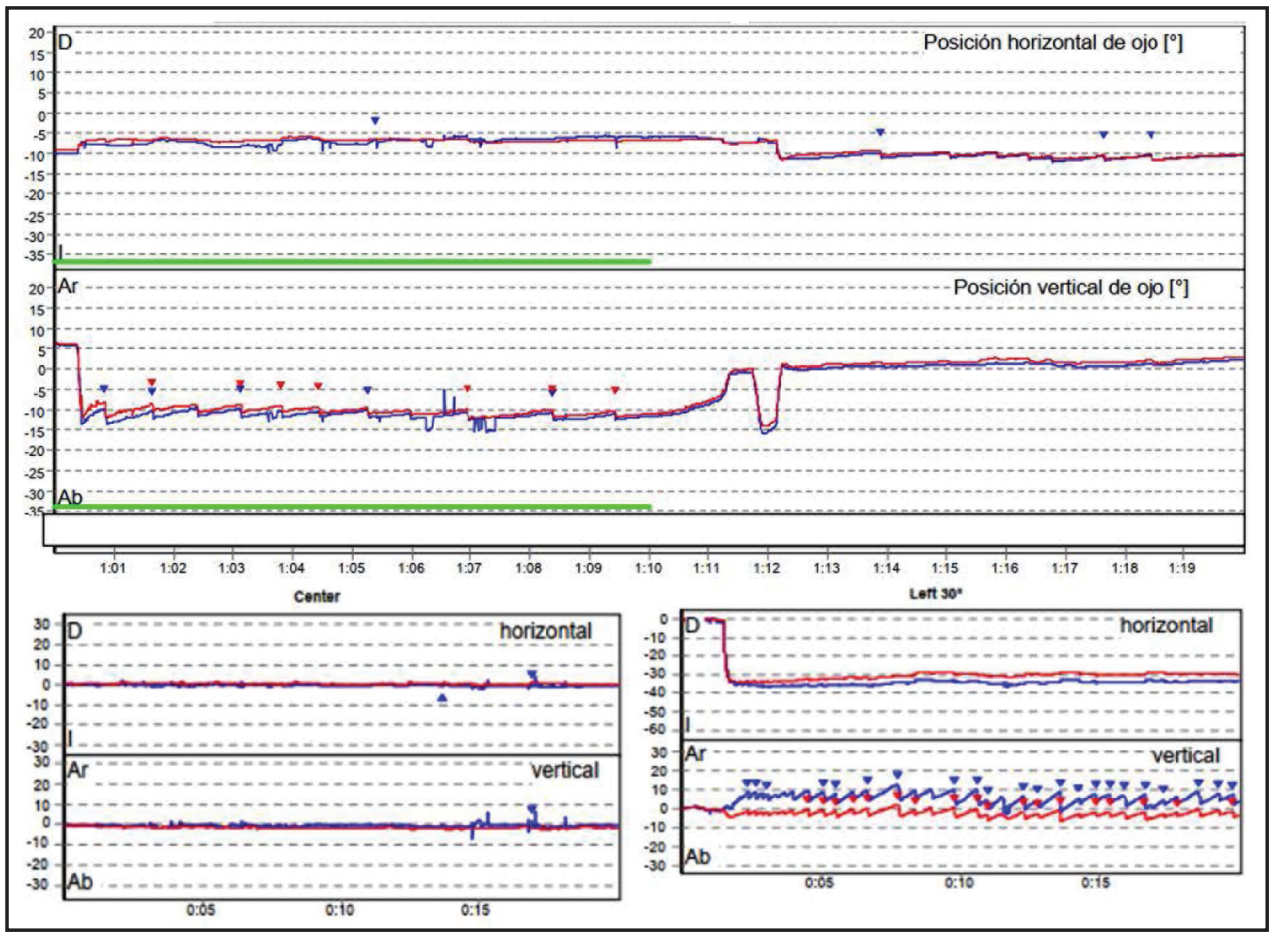

Figura 2. Video-oculografía caso clínico 1. Paneles superiores: registro en posición neutra de los ojos donde se observa la clara disminución del nistagmo al retirar la fijación ocular (desde la marca 1:10). Paneles inferiores: aumento de la intensidad del nistagmo con la mirada a izquierda en $30^{\circ}$ al comparar con mirada central. $D=$ derecha, $I=$ izquierda, $A r=a r r i b a, A b=a b a j o$.

recha e izquierda (Figura 3). Ante estos resultados, se solicita estudio imagenológico inicialmente con resonancia magnética de cerebro con contraste que la paciente no puede realizarse por claustrofobia. La paciente abandona sus controles médicos hasta la fecha.

\section{DISCUSIÓN}

Presentamos dos casos clínicos de nistagmo vertical hacia abajo atendidos en el Servicio de Otorrinolaringología del Hospital Clínico de la Universidad de Chile. En el primer caso se presenta un paciente con antecedentes cardiovasculares con estudio imagenológico compatible con secuela isquémica cerebelosa como causa de su DBN. El segundo caso no presenta antecedentes en su historia clínica que hagan sospechar una causa secundaria, pero esta no puede ser descartada hasta la fecha. Independiente de la causa, en todos los casos se presentan síntomas crónicos de similares características. Cabe destacar que, si bien en nuestros casos se observa en el examen físico un DBN con claridad, es de importancia realizar un estudio con videonistagmografía que permite objetivar dicho nistagmo y cuantifica su intensidad mediante la velocidad de la componente lenta (VCL) en las distintas posiciones.

Dentro de las causas de DBN se incluye patología cerebelosa o de tronco encefálico de diversa naturaleza que deben ser estudiadas mediante imágenes, idealmente resonancia magnética cerebral con énfasis en fosa posterior y contrastada con gadolinio. Esta patología puede ser²: neurodegenerativa (ataxias espinocerebelosas, atrofia multisistémica con compromiso cerebeloso, etc.), vascular de territorio posterior (compromiso de arterias vertebrales, basilar y cerebelosas, microangiopatía y malformaciones arteriovenosas), 


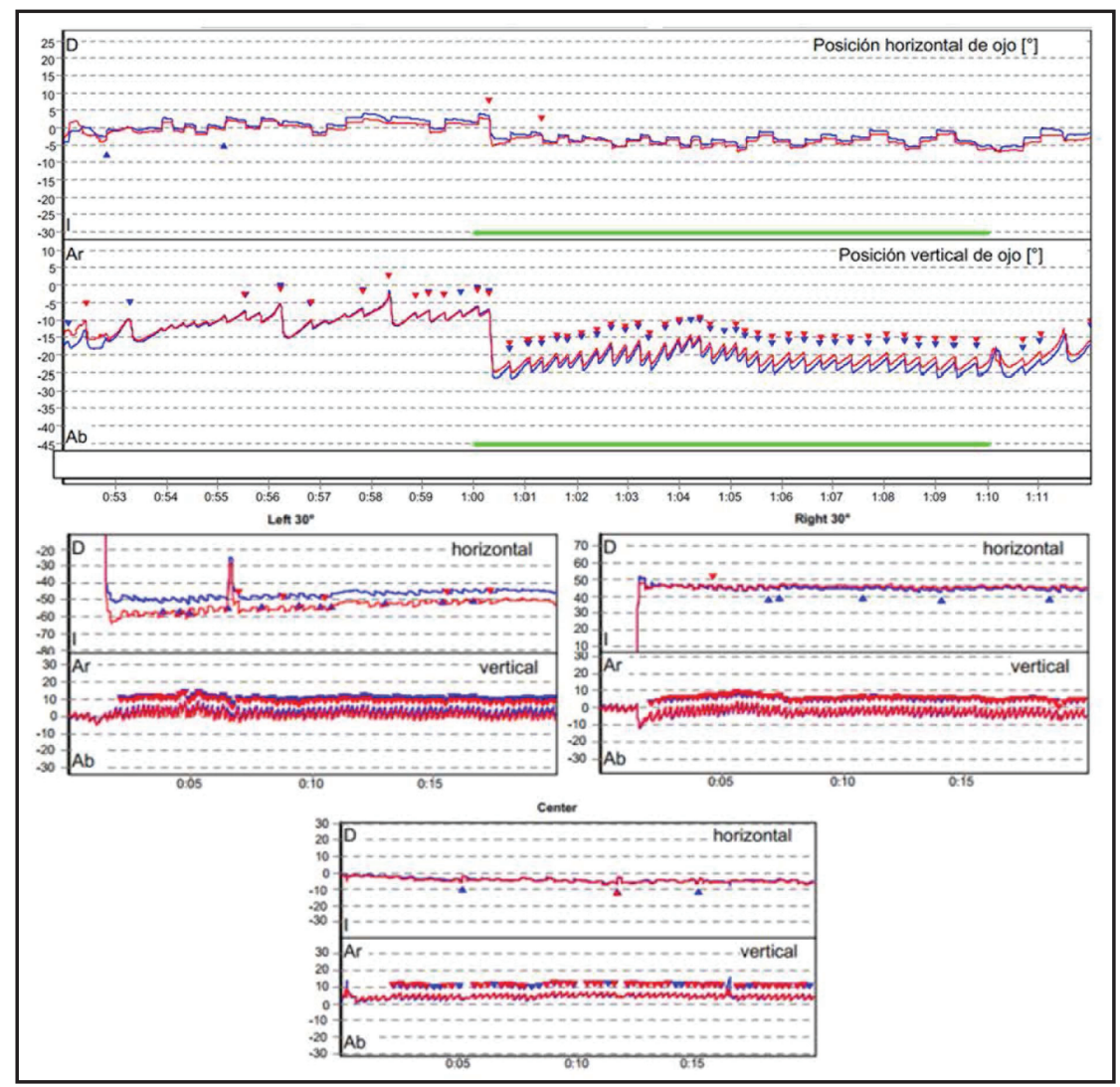

Figura 3. Video-oculografía caso clínico 2. Paneles superiores: registro en posición neutra de los ojos que evidencia aumento del nistagmo con fijación ocular (desde marca 1:00). Paneles inferiores: se observa aumento del nistagmo tanto en mirada $30^{\circ}$ izquierda y derecha en comparación a mirada central (center, panel más inferior). $D$ =derecha, $I$ =izquierda, $A r=a r r i b a, A b=a b a j o$.

malformaciones (Arnold-Chiari tipo I y II), toxicidad (carbamazepina, amiodarona, anticonvulsivantes, uso crónico de alcohol y litio $\left.{ }^{5,9,10}\right)$. En la terapia con litio es importante destacar que su rango terapéutico es estrecho y los síntomas de toxicidad pueden aparecer incluso dentro de rangos normales. En estos casos el DBN puede acompañarse de paresia en mirada horizontal y en su histopatología se describe pérdida de neuronas en núcleos integradores de la mirada horizontal y que conectan con estructuras encargadas de los movimientos oculares verticales, tales como núcleos prepósito hipogloso y núcleos vestibulares mediales ${ }^{10}$. Otras causas de DBN son neoplasias (primario, metástasis o síndrome paraneoplásico), enfermedades desmielinizantes (esclerosis múltiple) $)^{3,5}$, trauma, hipovitaminosis B12, migraña vestibular, enfer- medad de Ménière bilateral ${ }^{11}$ (causa rara de DBN), enfermedades genéticas (ataxia episódica tipo 2) y autoinmunes (anticuerpos antidescarboxilasa de ácido glutámico [anti-GAD]).

Con respecto al estudio imagenológico de estos pacientes, se describe en la literatura que hasta $50 \%$ de ellos presentará anormalidades en los estudios que pueden explicar su cuadro clínico, mientras los pacientes restantes tendrán estudio negativo para causas de DBN ${ }^{1}$. Actualmente existe escaso conocimiento respecto al pronóstico de esta patología. Wagner y cols realizó un estudio de seguimiento a 6 años de siete pacientes con DBN idiopático con mediciones de la VCL basal y subsecuentemente a los 2, 3 y 6 años sin encontrar cambios significativos en la VCL ni en su dependencia a la posición de la mirada. Los autores plantean que 
el DBN idiopático sería una patología de progresión lenta o representaría una etapa final, clínicamente aparente, de un DBN subclínico ${ }^{12}$.

Las medidas terapéuticas para este cuadro las podemos clasificar en: medidas generales, rehabilitación vestibular, tratamiento farmacológico y tratamiento quirúrgico ${ }^{1}$. Las medidas generales consisten en evitar agravantes como el decúbito, se sugiere descanso en posición semivertical ${ }^{13} \mathrm{y}$ en oscuridad $^{14}$; en nuestros casos nosotros recomendamos el descanso en posición semisentada para aminorar síntomas. La terapia de rehabilitación vestibular, especialmente ejercicios oculomotores y estímulos optokinéticos, ha demostrado ser de utilidad para el alivio sintomático de estos pacientes, pero no disminuye la $\mathrm{VCL}^{1}$; nuestro primer caso recibió dicha terapia con buena respuesta sintomática, como se describe en la literatura. La terapia farmacológica logra una mejoría tanto sintomática como disminución de la $\mathrm{VCL}^{1}$ y sus principales exponentes son las aminopiridinas (4-aminopiridina [4-AP] y 3,4-diaminopiridina $[3,4-D A P])^{1,3}$ que corresponden a bloqueadores no selectivos de canales de potasio voltaje-dependientes logrando un aumento de la actividad gabaérgica de las neuronas de Purkinje. Otra molécula que ha sido utilizada para el tratamiento del DBN es el baclofeno, pero se describe que hasta un tercio de los pacientes no responderán a este medicamento ${ }^{1}$. En nuestros casos no fueron indicadas las aminopiridinas debido a su alto costo.

Como última línea es posible recurrir a cirugía a nivel de los músculos extraoculares, como la tenotomía, para controlar los movimientos oculares anómalos. Hertle y col, realizaron una resección de $5 \mathrm{~mm}$ en ambos rectos superiores asociado a miectomía bilateral del oblicuo inferior en 17 pacientes, tanto niños como adultos, que no respondieron a tratamiento médico logrando mejoría significativa en oscilopsia subjetiva, agudeza visual binocular, desviación estrábica de la posición pri- maria, postura anómala de la cabeza, sensibilidad al contraste y en VCL, resultados que son aún más relevantes al considerar que aproximadamente solo $50 \%$ de los pacientes responderán a la terapia médica ${ }^{15}$ que además es de alto costo.

Algunas alternativas terapéuticas nuevas que están actualmente en fase de estudio son el feedback visual en tiempo real por computadora estudiado por el grupo de Teufel y cols ${ }^{16}$ que busca compensar el movimiento ocular causante de la oscilopsia de una forma no invasiva (sin anillos corneales ni lentes de contacto) y que ha demostrado una mejoría de la VCL en miradas laterales; y los estudios de Feil y cols ${ }^{4}$ con respecto al uso de clorzoxazona como una alternativa farmacológica con la que han observado mejoría en los parámetros de VCL, agudeza visual y posturografía en sus pacientes. En su estudio utilizaron $500 \mathrm{mg}$ de clorzoxazona tres veces al día, con una mejoría clínica significativa respecto al basal, pero menor a la terapia con 3,4-DAP. Así, el manejo de la sintomatología clínica del DBN debe ser planteada en forma individualizada, paciente a paciente, valorando las medidas de rehabilitación optokinética, terapia farmacológica y eventualmente quirúrgica.

\section{CONCLUSIÓN}

El DBN representa una entidad que el otorrinolaringólogo debe identificar y manejar en forma multidisciplinaria, incluyendo a neurólogos 0 neurocirujanos según corresponda. El estudio del paciente con DBN debe incluir videonistagmografía y resonancia magnética cerebral con énfasis en fosa posterior, ya que en la mayoría de los casos refleja una patología del sistema nervioso central. Ante el elevado costo de las aminopiridinas en nuestro medio, la rehabilitación oculomotora adquiere un papel fundamental en mejorar la calidad de vida de nuestros pacientes. 


\section{BIBLIOGRAFÍA}

1. Sotomayor D, Breinbahuer H. Síndrome de nistagmo vertical hacia abajo: Reporte de 2 casos y revisión de la literatura. Rev Otorrinolaringol Cir Cabeza Cuello 2016; 76: 63-70.

2. Wagner JN, Glaser M, Brandt T, Strupp M. Downbeat nystagmus: aetiology and comorbidity in 117 patients. J Neurol Neurosurg Psychiatry 2008; 79: 6727.

3. Helmchen C, Kirchroff JB, Göttlich M, Sprenger A. Postural Ataxia in Cerebellar Downbeat Nystagmus: Its Relation to Visual, Proprioceptive and Vestibular Signals and Cerebellar Atrophy. PLOS One 2017 5; 12: e0168808.

4. Feil K, Claassen J, Bardins S, Teufel J, Krafczyk S, Schneider E, Schinepp R, Jahn K, Kalla R, Strupp $M$. Effect of chlorzoxazone in patients with downbeat nystagmus: A pilot trial. Neurology 2013; 81: 1152-8.

5. Nakamagoe K, Fujizuka N, Koganezama T, Yamaguch T, TАМАоКА A. Downbeat nystagmus associated with damage to the medial longitudinal fasciculus of the pons: a vestibular balance control mechanism via the lower brainstem paramedian tract neurons. J Neurol Sci 2013; 328: 98-101.

6. Saito T, Aizawa H, Sawada J, Katayama T, Hasebe $\mathrm{N}$. Lesion of the nucleus intercalatus in primary position upbeat nystagmus. Arch Neurol 2010; 67: 1403-4.

7. Nakamagoe K, Shimizu K, Koganezawa T, Tamaoka A. Downbeat nystagmus due to a paramedian medullary lesion. J Clin Neurosci2012; 19: 1597-9.

8. Leigh RJ, Zee DS. The Neurology of Eye
Movements. Oxford University Press; Edición 4, 2006.

9. Schein F, Manoli P, Cathébras P. Lithium-induced downbeat nystagmus. Am J Ophthalmol Case Rep 2017; 7: 74-5.

10. JøRgensen JS, Landschoff Lassen L, Wegener M. LithiumInduced Downbeat Nystagmus and Horizontal Gaze Palsy. Open Ophthalmol J 2016; 10: 126-8.

11. Lee SU, Kim HJ, Lee ES, Chol JY, KIm JS. Ictal downbeat nystagmus in bilateral Meniere's disease. J Neurol 2017; 264: 2024-6.

12. Wagner J, Lehnen N, Glasauer S, Strupp M, Brandt T. Prognosis of idiopathic downbeat nystagmus. Ann N Y Acad Sci 2009; 1164: 479-81.

13. Spiegel R, Kalla R, Rettinger N, Schneider E, Straumann D, Marti S, Glasauer S, Brandt T, StRUPP M. Head position during resting modifies spontaneous daytime decrease of downbeat nystagmus. Neurology 2010; 75: 1928-32.

14. Spiegel R, Claassen J, Teufel J, Bardins S, Schneider E, Lehrer Rettinger N, Jahn K, da Silva Fa, Hahn A, Farahmand P, Brandt T, Strupp M, KALLA R. Resting in darkness improves downbeat nystagmus: evidence from an observational study. Ann N Y Acad Sci 2016; 1375: 66-73.

15. Hertle RW, Ahmad A. Clinical and electrophysiological results of eye muscle surgery in 17 patients with downbeat nystagmus. Indian J Ophthalmol 2019; 67: 109-15.

16. Teufel J, Bardins S, Spiegel R, Kremmyda 0 , Schneider E, Strupp M, Kalla R. Real-time computer-based visual feedback improves visual acuity in downbeat nystagmus - a pilot study. $J$ Neuroeng Rehabil 2016; 13: 1. 